\title{
Analysis of Rain Erosion of Coated Materials
}

\author{
George S. Springer, Cheng-I. Yang and Poul S. Larsen* \\ Department of Mechanical Engineering \\ The University of Michigan \\ Ann Arbor, Michigan 48105
}

(Received May 25, 1974)

\begin{abstract}
The behavior of coat-substrate systems subjected to repeated impingements of liquid droplets was investigated. The systems studied consisted of a thick homogeneous substrate covered by a single layer of homogeneous coating of arbitrary thickness. Based on the uniaxial stress wave model, the variations of the stresses with time were determined both in the coating and in the substrate. Employing fatigue theorems; algebraic equations were derived which describe the incubation period and the mass loss of the coating past the incubation period, in terms of the properties of the droplet, the coating and the substrate. The results were compared to available experimental data and good agreement was found between the present analytical results and the data.
\end{abstract}

\section{INTRODUCTION}

COMPONENTS OF HIGH speed aircraft and missiles may experience heavy damage when subjected to repeated impingements of rain droplets. The damage to nonmetallic components may be particularly severe. To protect such surfaces from rain erosion, these surfaces are frequently covered with a thin layer of coating.

The majority of the previous studies of rain erosion of coated materials have been experimental in nature (e.g., see References 1-6). These experimental investigations provide information on the behavior of a given coat-substrate combination under a given condition, but do not describe material behavior beyond the range of the experiments in which they were obtained. For the selection of the proper materials and for the design of the appropriate structures an analytical or semiempirical model would be needed, which would describe the response of coat. substrate systems in terms of the relevant parameters. These parameters should include the properties of the coating and the substrate, the thickness of the coating,

*Afdelingen for Fluid Mekanik, Den Polytekniske Laeteanstalt, Bygning 404, 2800 Lyngby, Denmark. 
and the impact velocity and size of the droplet. In recent years, progress towards this goal has been made by Morris [7], Engel and Piekutowski [8] and by Conn and his coworkers [9-11], who analyzed the stress history in various coat-substrate systems. Although the results of these investigations further our understanding of the processes which contribute to the failure of the coating and the substrate, as yet they are not capable of correlating fully the existing data.

The objective of this investigation was to develop a model which is consistent with experimental observation and which predicts quantitatively "erosion" of coated materials under previously untested conditions. In particular, the model proposed here is aimed at describing a) the "incubation period", i.e. the time elapsed before the mass loss of the coating becomes appreciable, and $b$ ) the degradation of the coating past the incubation period, as manifested by its mass loss. The model is based on fatigue concepts (e.g. References 12, 13), and is along the lines developed previously for homogeneous (uncoated) materials [13] .

\section{THE PROBLEM}

The problem investigated is the following. Spherical liquid droplets impinge repeatedly upon a plane, semi-infinite material consisting of a homogeneous substrate covered by a homogeneous coating (Figure 1). The thickness of the coating is $h$. The substrate is taken to be semi-infinite normal to the plane of the surface ( $x$ direction in Figure 1). The coating and the substrate are characterized by the following properties: density $\rho$, speed of sound $C$, modulus of elasticity $E$, Poisson's ratio $\nu$, ultimate tensile strength $\sigma_{u}$ and endurance limit $\sigma_{I}$. Parameters related to the coating and the substrate are denoted by $c$ and $s$, respectively. Parameters related to the droplet are identified by the subscript $L$. A perfect bond is assumed between the coating and the substrate, i.e. at the interface $(x=h)$ the stresses and the displacements are the same in the coating and the substrate.

The diameter of the droplets $d$, the angle of incidence $\theta$, and the velocity of impact $V$ are taken to be constant. The spatial distribution of the droplets is considered to be uniform. Accordingly, the number of droplets impinging on unit area in time $t$ is [13]

$$
h=\frac{6}{\pi} \frac{\left(V^{\prime} \cos \theta\right) I}{V_{t} d^{3}} t
$$

where $V_{t}$ is the terminal velocity, and $I$ is the rain intensity. The impingement rate is assumed to be sufficiently low so that all the effects produced by the impact of one droplet diminish before the impact of the next droplet [14].

The pressure within the droplet varies both with position and with time. For simplicity, the pressure at the liquid-surface interface is taken to be constant, its value being given by the water hammer pressure [15] 


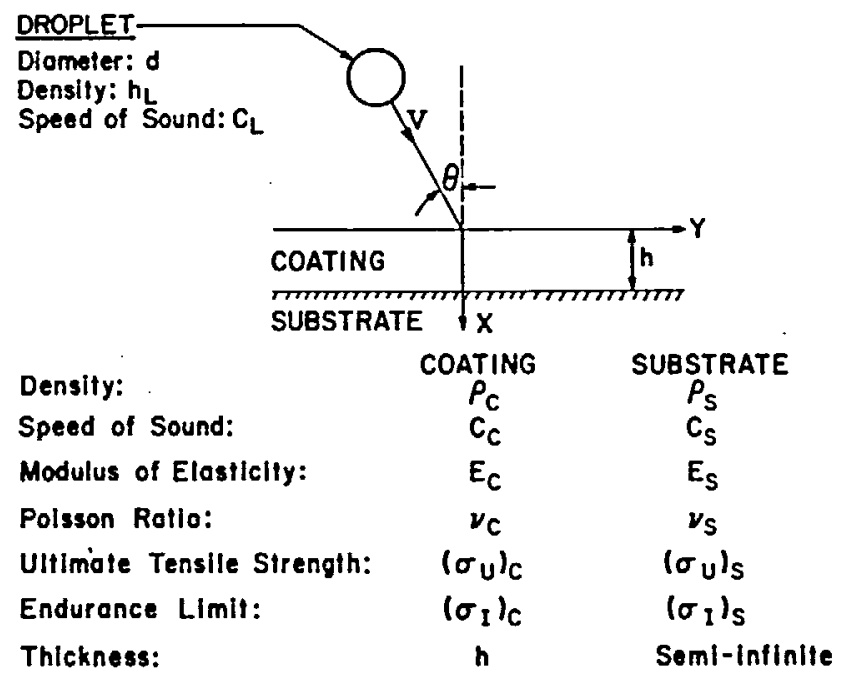

Figure 1. Droplet Jmpingement on a Coat-Substrate System. Description of Problem.

$$
P=\frac{\rho_{L} C_{L} V \cos \theta}{1+\frac{\rho_{L} C_{L}}{\rho_{c} C_{c}}}
$$

Although more accurate representation of the pressure is possible [15] the accuracies afforded by the use of Equation (2) will suffice in the present analysis. The duration of the pressure at the interface is approximated by

$$
t_{L}=\frac{2 d}{C_{L}}
$$

The forces, created by the repeated droplet impacts, damage the material as manifested by the formation of pits and cracks on the surface, and by weight loss of the coating material. Experimental evidence indicates that under a wide range of conditions the weight loss $W$ varies with time $t$ as shown, schematically, in Figure 2a. For some period of time, referred to as incubation period, the weight loss is insignificant. Between the end of the incubation period $t_{i}$ and a time denoted by $t_{f}$ the weight loss varies nearly linearly with time. After $t_{f}$ the relationship between $I V$ and $t$ becomes more complex. Here, we will be concerned only with the behavior of the material up to time $t_{f}$. In most practical situations the usefulness of the material does not extend beyond $t_{f}$. 


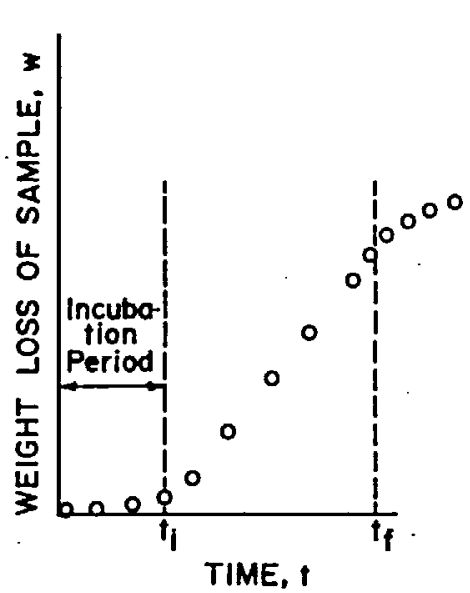

Figure 2a. Schematic of the Experimentol Results.

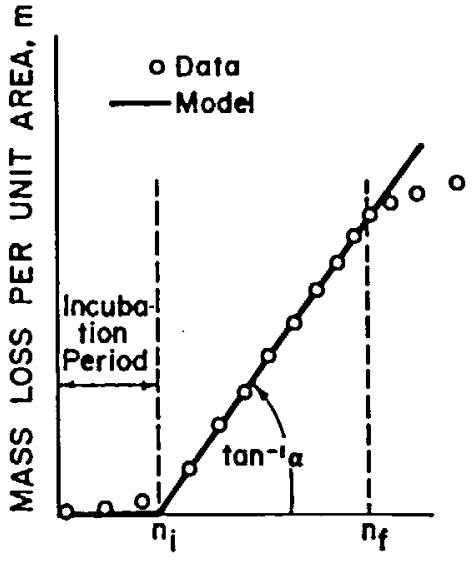

NUMBER OF IMPACTS PER UNIT AREA, $n$

Figure 2b. The Solution Model.

It is advantageous to replace the total weight loss of the sample by the mass loss per unit area $m$, and the time by the number of droplets impinging upon unit area $n$. It is now assumed that the data can be approximated by two straight lines as shown in Figure $2 b$, i.e.

$$
\begin{array}{ll}
m=0 & 0<n_{i} \\
m=\alpha\left(n-n_{i}\right) & n_{i}<n<n_{f}
\end{array}
$$

Thus, the material loss $m$ produced by a certain number of impacts $n$, can be calculated once the incubation period $n_{i}$ and the rate of subsequent mass loss (as characterized by the slope $\alpha$ ) are known. This model is valid provided there is an incubation period.

In order to establish $n_{i}$ and $\alpha$, the stress history in the coating must be known. Thus, first expressions are derived which describe, in suitable form, the variation of the stress with time in the coating and in the substrate.

\section{STRESS HISTORY OF THE COATING AND THE SUBSTRATE}

The stress waves propagating through the coating and the substrate are considered to be one dimensional, propagating normal to the surface. Waves parallel to the surface (shear waves) are neglected (Figure 3). The magnitude of the initial stress wave, denoted by $\sigma_{1}$, is identical to the hydrostatic pressure $P$, i.e.,

$$
\sigma_{1}=P
$$



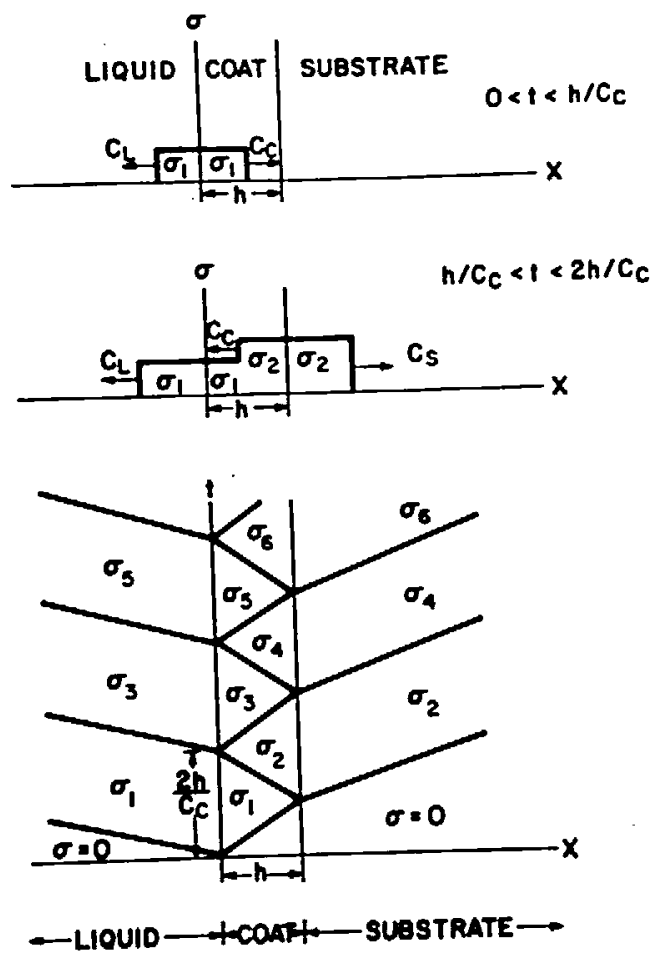

Figure 3. Stress W'ave Pattern in the Coating and in the Substrate.

$P$ is given by Equation (2). At the coat-substrate interface a portion of the stress wave is transmitted into the substrate while a portion of it is reflected back into the coating. Thus, there is a sequence of "left" traveling waves in the coating of magnitude $\sigma_{2 k}[16-17]$

$$
\frac{\sigma_{2 k}}{\sigma_{1}}=\frac{1+\psi_{s c}}{1-\psi_{s c} \psi_{L c}}\left[1-\left(\psi_{s c} \psi_{L c}\right)^{k}\right]
$$

After the time interval $t=C_{c} / 2 h$ a "left" traveling wave reaches the coat-liquid interface and a new "right" traveling wave, of magnitude $\sigma_{2 k-1}$ is generated at the $x=0$ surface $[16-17]$

$$
\frac{\sigma_{2 k}-1}{\sigma_{1}}=\frac{\sigma_{2 k}}{\sigma_{1}}-\psi_{s c}\left(\psi_{s c} \psi_{L c}\right)^{k-1}
$$

where $k$ is an integer $(k=1,2,3 \ldots)$ and 


$$
\psi_{s c}=\frac{Z_{s}-Z_{c}}{Z_{s}+Z_{c}} \quad \psi_{L c}=\frac{Z_{L}-Z_{c}}{Z_{L}+Z_{c}}
$$

$Z$ is the impedance of the material

$$
Z=\rho c
$$

Note that the stress history in the coating depends on the relative magnitudes of $Z_{L}, Z_{c}$ and $Z_{s}$. This is illustrated in Figure 4, where the variation of the stress with time is shown for the four possible combinations of impedances. After a long period of time (i.e. after a large number of reflections, $k \rightarrow \infty$ ) the stress at both on the surface of the coating $(x=0)$ and at the coat-substrate interface $(x=h)$ approaches the constant value

$$
\sigma_{\infty}=\sigma \lim _{k \rightarrow \infty} \sigma_{2 k}=\frac{1+\psi_{s c}}{1-\psi_{s c} \psi_{L c}}=\frac{1+\dot{Z}_{L} / Z_{c}}{1+Z_{L} / Z_{s}}
$$

$\sigma_{\infty}$ is the stress that would occur in the substrate if the droplet would impinge upon it directly in the absence of a coating [17]. It is evident from Figure 4 that the coating reduces the stresses in the substrate only if the appropriate coating material (i.e. appropriate combination of $Z_{L}, Z_{c}$ and $Z_{s}$ ) is selected (Figures $4 \mathrm{c}$ and $4 \mathrm{~d}$ ). For certain combinations of coating and substrate the mean stresses in the substrate are actually higher with the coating than without it (Figures $4 a$ and $4 b$ ). This result clearly indicates the importance of the proper selection of the material used as coating for a particular substrate.

Equations (6) and (7) describe the variation of the stress with time in the coating. For our further calculations it is convenient to replace the stepwise variation of the stress by a continuous function. To accomplish this Equation (6) is rewritten in the form

$$
\frac{\sigma_{2 k}}{\sigma_{1}}=\frac{\sigma_{\infty}}{\sigma_{1}}-\left(\frac{\sigma_{\infty}}{\sigma_{1}}-\frac{\sigma_{2}}{\sigma_{1}}\right)\left(\psi_{s c} \psi_{L c}\right)^{k-1}
$$

Equation (11) is now approximated by the expression

$$
\frac{\sigma_{2 k}}{\sigma_{1}}=\frac{\sigma_{\infty}}{\sigma_{1}}-\left(\frac{\sigma_{\infty}}{\sigma_{1}}-\frac{\sigma_{2}}{\sigma_{1}}\right) \exp \left(-\frac{k-1}{k_{e}}\right)
$$

By replacing Equation (11) by Equation (12) we replace, in effect, the stepwise stress function with an exponential curve, as illustrated in Figure 5. In Equation (12) $k_{e}$ is the number of reflections required for the stress to reach 63.3 percent of 

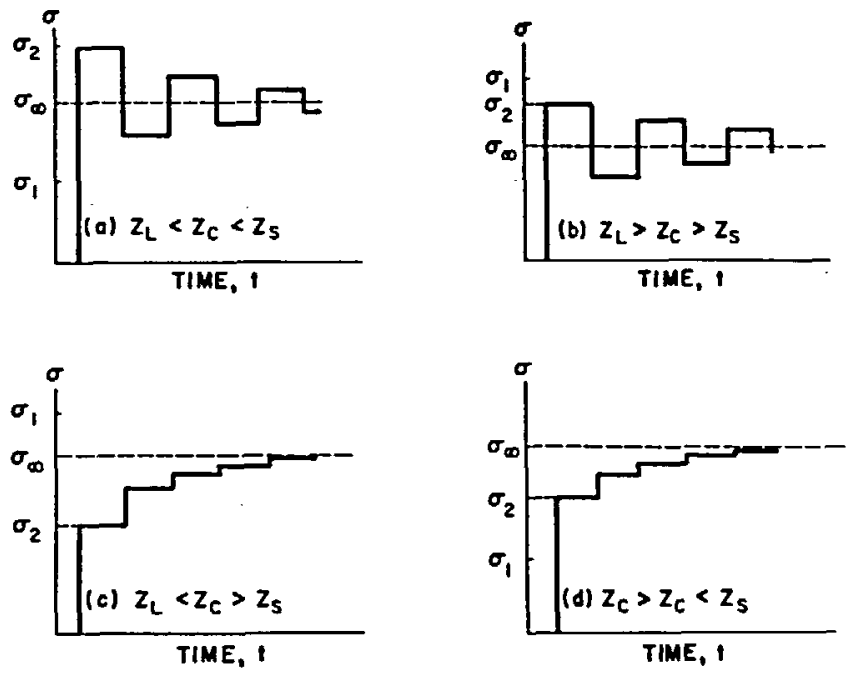

Figure 4. The Variation of the Stress at the Coat-Substrate Interface.

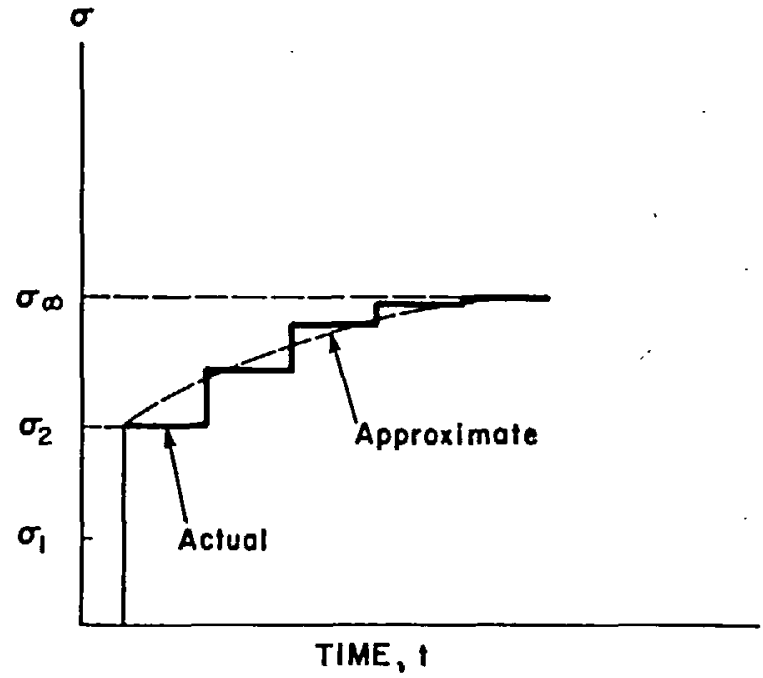

Figure 5. The Actual and Approximate Variation of the Stress at the Coat-Substrate Interface.

$\sigma_{\infty}$. To evaluate $k_{e}$ we introduce the condition that the area under the actual (stepwise) and the exponential curves are to be the same. This condition requires that the following equality be satisfied 


$$
\sum_{k=1}^{\infty} \cdot\left[\frac{\sigma_{\infty}}{\sigma_{1}}-\left(\frac{\sigma_{\infty}}{\sigma_{1}}-\frac{\sigma_{2}}{\sigma_{1}}\right)\left(\psi_{s c} \psi_{L c}\right)^{k-1}\right]=\int_{1}^{\infty}\left[\frac{\sigma_{\infty}}{\sigma_{1}}-\left(\frac{\sigma_{\infty}}{\sigma_{1}}-\frac{\sigma_{2}}{\sigma_{1}}\right) \exp \left(-\frac{k-1}{k_{e}}\right)\right] d k
$$

Evaluating the summation and the integral in Equation (13) we obtain

$$
k_{e}=\frac{1}{1-\psi_{s c} \psi_{L c}}
$$

Substitution of Equations (8) and (9) into Equation (14) yields

$$
k_{e}=\frac{1+Z_{L} / Z_{s}}{2} \frac{1+Z_{c} / Z_{s}}{1+Z_{L} / Z_{s}}
$$

In the absence of coating $Z_{s}=Z_{c}$ and $k_{e}=1$, which, as expected, shows that there are no reflections in a semi-infinite material.

The time required for $k_{e}$ number of reflections to occur is (see Figure 3)

$$
t_{e}=k_{e} \frac{2 h}{C_{c}}
$$

and the number of reflections during this time is

$$
k_{e}=t_{e} \frac{C_{c}}{2 h}
$$

Similarly, the number of reflections which occur during the duration of the impact $t_{L}$ (given by Equation 3 ) is

$$
k_{L}=t_{L} \frac{C_{c}}{2 h}=\frac{C_{c}}{C_{L}} \frac{d}{h}
$$

It is to be noted that $k_{e}$ is independent of the thickness of the coating (see Equation 15), while $k_{L}$ depends on $h$. For thick coating $(h / d \rightarrow \infty) k_{L} \rightarrow 0$ and for thin coating $(h / d \rightarrow 0) k_{L} \rightarrow \infty$. Thus, the ratio

$$
\gamma=\frac{k_{L}}{k_{e}}
$$

may vary between zero and infinity. It is convenient to bridge these two limits by the exponential curve 


$$
\bar{k}=k_{e}\left[1-\exp \left(-\frac{k_{L}}{k_{e}}\right)\right]=k_{e}[1-\exp (-\gamma)]
$$

$\bar{k}$ represents the average number of reflections in the coating. The variation of $\bar{k}$ with $\gamma$ is illustrated in Figure 6. For thick coating $\bar{k}$ becomes

$$
\bar{k}_{h / d \rightarrow \infty}=0
$$

For thin coating Equation (20) reduces to

$$
\bar{k}_{h / d \rightarrow o}=k_{e}
$$

which is, by our definition, the maximum number of reflections which may occur in the coating.

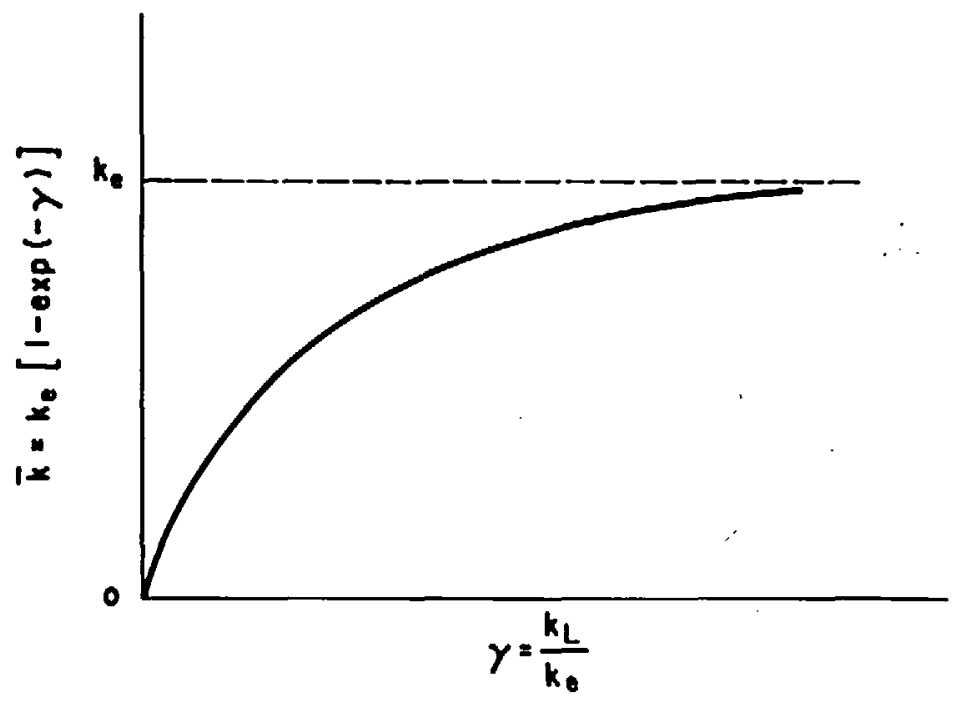

Figure 6. The Variation of the Number of Stress Wave Reflections in the Coating with $\gamma$.

We may evaluate now the average values of the stresses at the coat-liquid $(x=0)$ and at the coat-substrate interfaces $(x=h)$ during the period of impact $n_{L}$. The average stress at $x=0$ is

$$
\bar{\sigma}^{o}=\frac{1}{k_{L}} \stackrel{k_{L}}{k^{\Sigma}}{ }_{1} \sigma_{2 k-1}
$$

and at $x=h$ is 


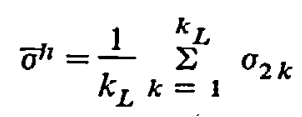

Substituting Equations (6), (7) and (10) into Equations (23) and (24) and utilizing the exponential approximation given by Equation (12), we obtain

$$
\begin{gathered}
\frac{\bar{\sigma}^{o}}{\sigma_{1}}=\frac{1+\psi_{s c}}{1-\psi_{s c} \psi_{L c}}\left[1-\psi_{s c} \frac{1+\psi_{L c}}{1+\psi_{s c}} \frac{1-\exp (-\gamma)}{\gamma}\right] \\
\frac{\bar{\sigma}^{n}}{. \sigma_{1}}=\frac{1+\psi_{s c}}{1-\psi_{s c} \psi_{L c}}\left[1-\psi_{s c} \psi_{L c} \frac{1-\exp (-\gamma)}{\gamma}\right]
\end{gathered}
$$

If the coating is of the same material as the substrate $\psi_{s c}=0$ and Equation (25a) reduces to

$$
\bar{\sigma}^{o}=\sigma_{1}=P
$$

The force exerted by the droplet on the surface of the coating also varies with time. The average force on the surface during the duration of one impact $t_{L}$ is

$$
\bar{F}=\bar{\sigma}^{\circ} \frac{\pi d^{2}}{4}
$$

The foregoing equations describe the stress history in the coating and in the substrate when the substrate is covered by a single layer of coating. The results could be generalized readily to include two or more layers of coatings. It is emphasized, however, that the expressions here developed are not restricted to thin coatings, but may be applied to coatings of arbitrary thicknesses. The thickness of the coating enters the results through the parameter $\gamma$. From Equations (15), (18) and (19) we have

$$
\gamma=\frac{C_{c}}{C_{L}} \frac{d}{h}\left(\frac{1+Z_{L} / Z_{s}}{1+Z_{c} / Z_{s}}\right)\left(\frac{1+Z_{L} / Z_{s}}{2}\right)
$$

For a thick coating $(h / d \rightarrow \infty) \gamma$ becomes

$$
\gamma_{h / d \rightarrow \infty}=0
$$

For a thin coating $(h / d \rightarrow 0) \gamma$ assumes the value 


$$
\gamma_{h / d \rightarrow 0}=\infty
$$

\section{INCUBATION PERIOD $n_{i}$}

It has been recognized in the past that fatigue plays an important role in the erosion process $[12,14,18-23]$, particularly in the "early" stages of the process, corresponding to the incubation period. Applying fatigue concepts to the problem of rain erosion, Springer and Baxi [13] recently established a semiempirical formula which describes the incubation period in a homogeneous material. Here, Springer and Baxi's analysis is extended to homogeneous materials covered by a single layer of coating. The analysis is based on the concept that fatigue theorems established for the torsion and bending of bars might be applied, at least qualita. tively, to materials subjected to repeated liquid impingement. The failures of bars undergoing repeated torsion or bending have been found to follow Miner's rule [24]

$$
\frac{f_{1}}{N_{1}}+\frac{f_{2}}{N_{2}}+\ldots \frac{f_{q}}{N_{q}}=a_{1}
$$

where $f_{1}, f_{2} \ldots f_{q}$ represent the number of cycles the specimen is subjected to specified overstress levels $\sigma_{e l}, \sigma_{e 2} \ldots \sigma_{e q}$, and $N_{1}, N_{2} \ldots N_{q}$ represent the life (in cycles) at these overstress levels, as given by the fatigue ( $\sigma_{e}$ versus $\left.N\right)$ curve. $a_{1}$ is a constant.

Let us now consider a point $B$ on the surface of the material as shown in Figure 7. Each droplet impinging upon the surface creates a stress'at point $B$. Assuming that the force created by the droplet at its point of impact is a "point force", the stress at point $B$ due to any one droplet is [25]

$$
\sigma=\frac{\bar{F}\left(1-\nu_{c}\right)}{2 \pi r^{2}}
$$

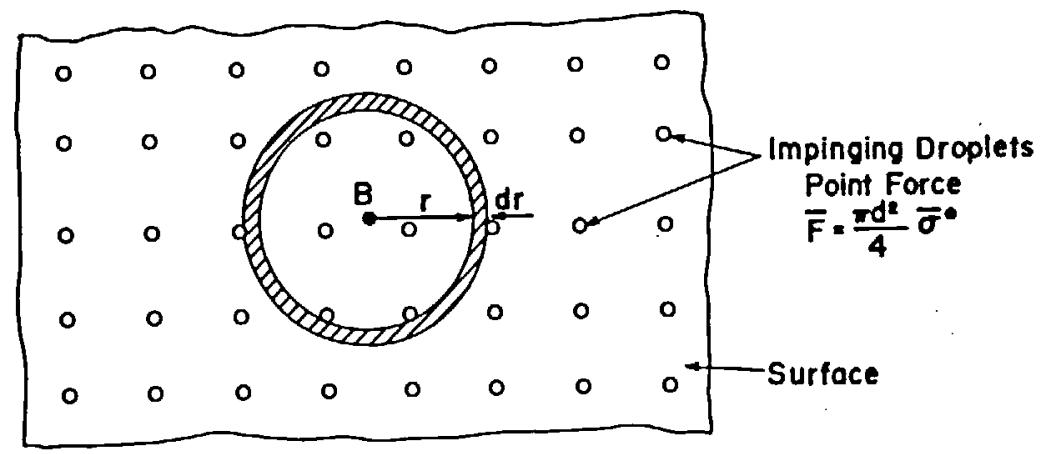

Figure 7. Force Distribution on the Surface of the Coating. 


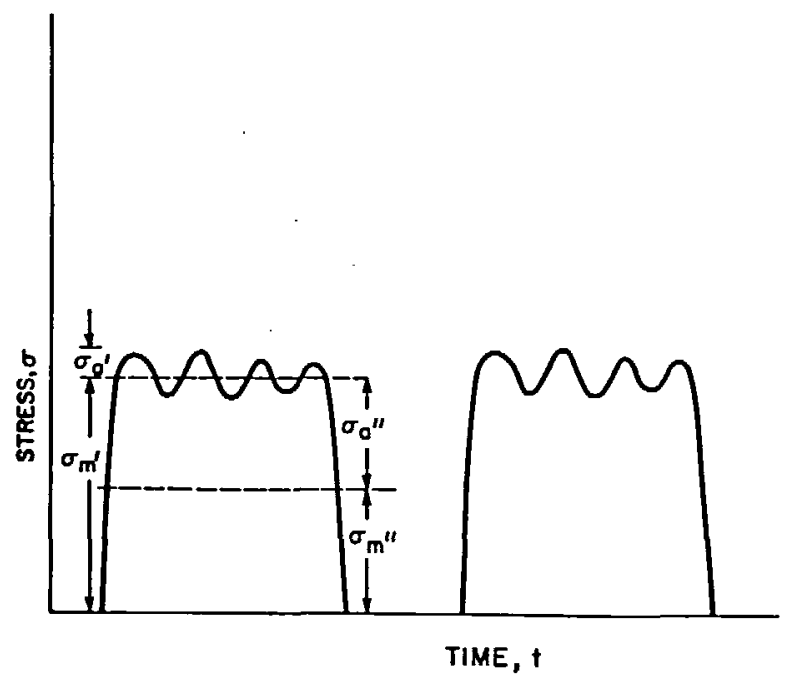

Figure 8. The Variation of the Stress with Time at the Liquid Droplet-Coat Interface.

where $\bar{F}$ is given by Equation (27). Due to the propagation and reflection of the stress waves in the coating (as discussed in the previous section) the stress in the coating does not remain constant, but fluctuates, as illustrated in Figure 8. Fatigue life of the material is generally calculated using an "equivalent dynamic stress" [26]

$$
\sigma_{e}=\frac{\sigma_{a} \sigma_{m}}{\sigma_{u}-\sigma_{m}}
$$

where $\sigma_{u}$ is the ultimate tensile strength of the material. In the present case $\sigma_{e}$ may be separated into two parts $\sigma_{e}=\sigma_{e}^{\prime}+\sigma_{e}^{\prime \prime}$. The first part, $\sigma_{e}^{\prime}$ is due to oscillations about the mean $\sigma_{m}^{\prime}=\sigma$ with amplitude $\sigma_{a}^{\prime}$. The second part $\sigma_{e}$ " is due to "oscillation" about the mean $\sigma_{m}^{\prime \prime}=\sigma / 2$, with a constant amplitude $\sigma_{a}^{\prime \prime}=\sigma / 2$. Thus, $\sigma_{a}^{\prime}$ is not a constant but varies with time. For simplicity, we assume that $\sigma_{a}^{\prime}$-is a constant with a value equivalent to the maximum amplitude, i.e.

$$
\sigma_{a}^{\prime}=\left|\sigma_{2}-\sigma\right|
$$

Equations (7) and (34) yield

$$
\sigma_{a}^{\prime}=\sigma\left|\psi_{s c}\right|
$$

The equivalent dynamic stresses corresponding to the two modes of stress oscillations just described may thus be written as 


$$
\sigma_{e}^{\prime}=\frac{\sigma\left|\psi_{s c}\right| \sigma_{u}}{\sigma_{u}-\sigma} \quad \sigma^{\prime \prime}=\frac{(\sigma / 2) \sigma_{u}}{\sigma_{u}-(\sigma / 2)}
$$

The number of cycles for which the material at point $B$ is subjected to a given stress between $\sigma_{e}$ and $\sigma_{e}+d \sigma_{e}$ is equal to the number of impacts on a $d r$ wide annulus located at $r$ (Figure 7). During the incubation period the total number of impacts on the annulus is

$$
f_{i}=n_{i} 2 \pi r d r
$$

For each single impact the number of stress oscillations in the coating is $\bar{k}$ (Equation 20). The total number of stress oscillations during $f$ impact is, therefore, $\bar{k} f_{i}$. Accordingly, Miner's rule becomes

$$
\sum_{i}\left(\frac{f_{i}}{N_{i}^{\prime}}+\frac{\bar{k} f_{i}}{N_{i}^{\prime \prime}}\right)=a_{1}
$$

where $N_{i}^{\prime}$ is the fatigue life for overstress levels at $\sigma_{e}^{\prime}$ and $N_{i}^{\prime \prime}$ is the fatigue life for overstress levels at $\sigma_{e}^{\prime \prime}$.

Since $r$ varies continuously from zero to infinity, Equations (37) and (38) may be written as

$$
\int_{0}^{\infty} \frac{n_{i} 2 \pi r}{N^{\prime}} d r+\int_{0}^{\infty} \frac{\bar{k} n_{i} 2 \pi r}{N^{\prime \prime}} d r=a_{1}
$$

The first term on the left hand side represents the stress oscillation about $\sigma_{m}=\sigma / 2$ and the second term the oscillation about $\sigma_{m}=\sigma$. From Equation (32) $r d r$ is

$$
r d r=-\frac{1}{2 \pi} \frac{\bar{F}\left(1-2 \nu_{c}\right)}{2 \sigma^{2}} d \sigma
$$

Equation (39) can thus be written as

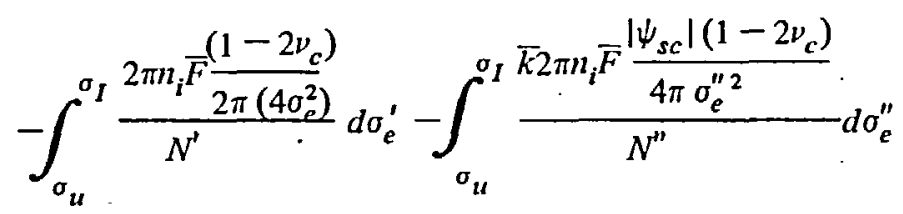

The lower and upper limits of the integrals have been changed to the ultimate tensile strength $\sigma_{u}$ and the endurance limit $\sigma_{l}$, respectively. In order to perform the 
integration the fatigue life $N$ must be known as a function of the stress $\sigma_{e}$. For most materials the fatigue curve between $\sigma_{u}$ and $\sigma_{I}$ may be approximated by [13]

$$
\begin{gathered}
N=\left(\sigma_{u} / \sigma_{e}\right)^{b} \\
\log _{10}\left(\frac{b_{2}}{\sigma_{I_{c}}}\right) \\
\left(\frac{\sigma_{u_{c}}}{\sigma_{I_{c}}}\right)
\end{gathered}
$$

where $b_{2}$ corresponds to the "knee" in the fatigue curve. Substituting Equations (42) and (27) into Equation (41) and integrating we obtain

$$
\frac{\pi d^{2}}{4} n_{i} \bar{\sigma}^{o}\left(1-D_{c}\right) \frac{\sigma_{u_{c}}^{b-1}-\sigma_{I_{c}}^{b}-1}{4(b-1) \sigma_{u_{c}}^{b}}\left(1+2 \mid \psi_{s c} \bar{k}\right)=a_{1}
$$

As noted before, the subscript $c$ refers to the coating. Introducing the definitions

$$
\begin{aligned}
& S=\frac{4 \sigma_{u}(b-1)}{\left(1-2 v_{c}\right)\left[1-\left(\frac{\sigma_{I}}{\sigma_{u_{c}}}\right)^{b-1}\right]} \cong \frac{4\left(\sigma_{u_{c}}\right)(b-1)}{1-2 v_{c}} \\
& S_{e}=\frac{S}{1+2 \bar{k}\left|\psi_{s c}\right|} \\
& n_{i}^{*}=n_{i} \frac{\pi d^{2}}{4}
\end{aligned}
$$

Equation (44) becomes

$$
n_{i}^{*}=a_{1} \frac{S_{e}}{\bar{\sigma}^{o}}
$$

The parameter $S_{e}$ characterizes the "strength" of the material. Thus, the number of impacts needed to initiate damage is propositional to the ratio of the "strength" of the material $S_{e}$ to the stress $\bar{\sigma}^{o}$ produced by the impinging droplets. Such a dependence of $n_{i}^{*}$ on $S_{e}$ and $\tilde{\sigma}^{o}$ is reasonable, since the length of the incubation period is expected to increase with increasing $S_{e}$ and with decreasing $\sigma^{\circ}$. However, in view of the fact that Equation (48) is based on the fatigue properties of materials 
in pure torsion and bending, one cannot expect a linear relationship to hold between $n_{i}^{*}$ and $S_{e} / \bar{\sigma}^{o}$. In order to extend the range of applicability of Equation (48), while retaining its major feature (namely the functional dependence of $n_{i}^{*}$ on $\left.S_{e} / \bar{\sigma}^{\circ}\right)$ we write

$$
n_{i}^{*}=a_{1}\left(\frac{S_{e}}{\bar{\sigma} o}\right)^{a_{3}}=a_{1}\left[\frac{S}{\bar{\sigma}^{o}} \frac{1}{1+2 \bar{k}\left|\psi_{s c}\right|}\right]^{a_{2}}
$$

where both $a_{1}$ and $a_{2}$ are as yet undetermined constants.

For a homogeneous material (in the absence of coating) the incubation period is [13]

$$
n_{i_{H}}^{*}=a_{1}\left(\frac{S}{P}\right)^{a}
$$

Both $P$ and $\bar{\sigma}^{o}$ denote an average stress at the surface. Note that $n_{i}^{*}$ and $n_{i}^{*}$ differ only by the factor $1 /\left(1+2 \bar{k} \mid \psi_{s c}\right)$. This factor represents the damping effect of the coating.

A homogeneous material may be viewed as either a material with very thick coating $(h / d \rightarrow \infty, \bar{k} \rightarrow 0$, Equation 21), or one in which the coating and the substrate are made of the same material $\left(\psi_{s c}=0\right.$, Equation 8$)$. It is evident that for either one of these conditions Equation (49) reduces to Equation (50), provided that the constants $a_{1}$ and $a_{2}$ have the appropriate values. To ensure that in the limits $\left(\bar{k} \rightarrow 0\right.$ and/or $\left.\psi_{s c} \rightarrow 0\right)$ Equations (49) and (50) become equal we adopt here the same values for $a_{1}$ and $a_{2}$ as were derived by Springer and Baxi [13] for homogeneous materials.* Using the values $a_{1}=7.1 \times 10^{-6}$ and $a_{2}=5.7$ we obtain

$$
n_{i}^{*}=7.1 \times 1 \sigma^{6}\left(\frac{S_{e}}{\bar{\sigma}^{o}}\right)^{5.7}
$$

Equation (51) gives the incubation period of a single layer of coating of arbitrary thickness. The validity of the model must now be evaluated by comparing this result to experimental data. The comparison is presented in Figure 9. In this figure all the data are included for which both $n_{i}$ and the relevant material properties $\left(\sigma_{u}\right.$, $\left.\sigma_{I}, b_{2}, \nu, E, \rho\right)$ for both the coating and the substrate were available. As can be seen, there is excellent correlation between the model and the data, lending support to the validity of the model.

*The value for the constant $a_{1}$ was given in Reference 13 as $3.7 \times 10^{-4}$. This value was obtained by using the stress $\sigma$ instead of $\sigma_{e}$ in calculating the fatigue life. Whe $\sigma$ is replaced by $\sigma_{e} a_{1}$ becomes $7.1 \times 10^{-6}$ (see Reference 17 ). 


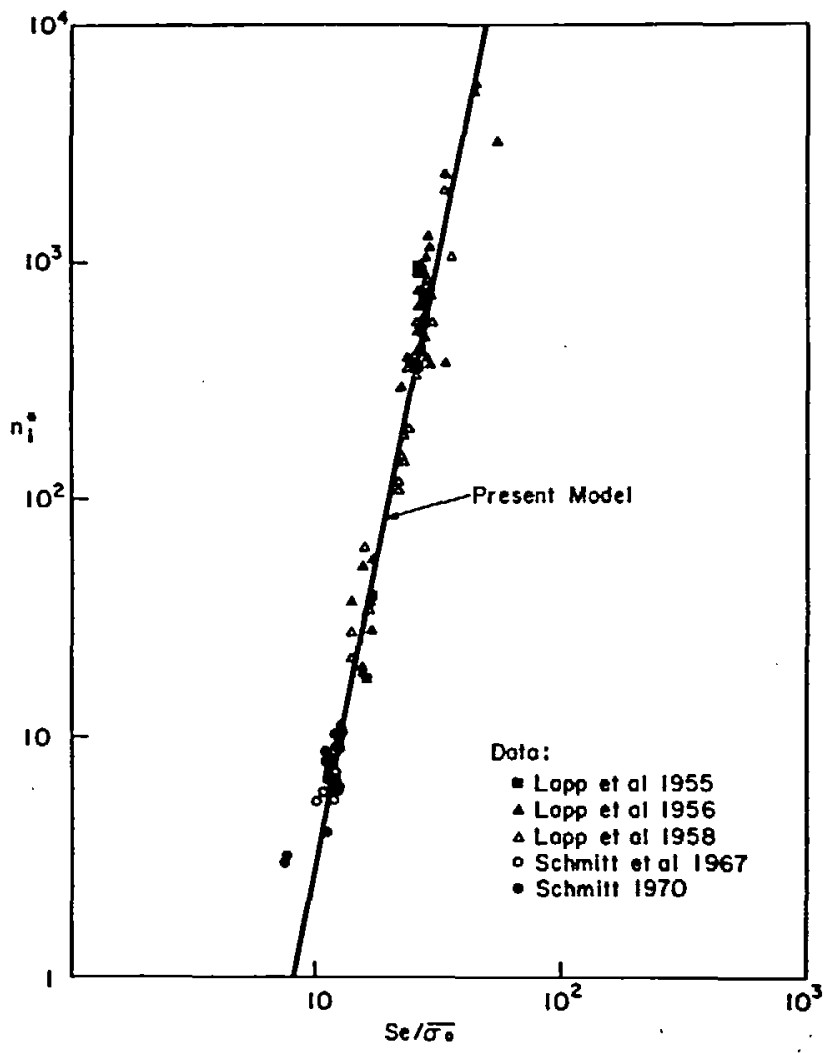

Figure 9. Incubation Period $n_{i}^{*}$ versus $S_{e}{ }_{\sigma^{0}}$. Solid Line: Model (Equation 51). Symbols Defined in Table 1.

\section{RATE OF MASS REMOVAL}

The mass removal rate of coat-substrate systems can be calculated in a manner analogously to the mass removal rate of homogeneous materials. It was shown in Reference 17 that the analysis and the results obtained for homogeneous materials (as presented in Reference 13) can be applied directly to coated materials. For homogeneous materials the dimensionless mass loss rate $\alpha^{*}$ was found to be [13]

$$
\alpha^{*}=a_{3} \frac{1}{\left(n_{i}^{*}\right)} a_{6}
$$

where $\alpha^{*}$ is defined as

$$
\alpha^{*}=\frac{\alpha}{\pi \rho d^{3} / 4}
$$


Table 1. Description of Data and Simbols Used in Figures 9, 10, and $11^{*}$

\begin{tabular}{|c|c|c|c|c|c|c|c|}
\hline $5 y=01$ & Inveselgator & Coating & Substrate & $\begin{array}{l}\text { Costing } \\
\text { Thickicss } \\
\text { - }\end{array}$ & $\begin{array}{l}\text { Velocity } \\
\text { ft/sec }\end{array}$ & $\begin{array}{l}\text { Intersity } \\
(\ln / \mathrm{hr})\end{array}$ & $D \cos \$ 120$ \\
\hline & \multirow[t]{2}{*}{$\begin{array}{c}\text { Lapp et } \\
\text { 1955 }\end{array}$} & Necprene & $\begin{array}{l}\text { Polyester } \\
\text { Aluednu } \\
\text { steel } \\
\text { Alu=inu }\end{array}$ & $\begin{array}{l}8.9,10 \\
15,20 \\
20 \\
5\end{array}$ & $\begin{array}{l}731 \\
877\end{array}$ & \multirow[t]{2}{*}{1} & \multirow[t]{2}{*}{1.9} \\
\hline & & Teflo: & Alusinte & 5.10 & 731 & & \\
\hline & \multirow[t]{3}{*}{$\begin{array}{l}\text { Lappet }=1 \\
1956 \cdot\end{array}$} & Neoprene. & $\begin{array}{l}\text { Polyester } \\
\text { Aluninua }\end{array}$ & $\begin{array}{l}4-5,7,10,20 \\
15-17\end{array}$ & $\begin{array}{l}877 \\
811 \\
\end{array}$ & \multirow[t]{3}{*}{1} & \multirow[t]{3}{*}{1.9} \\
\hline & & Polye thy lene & Polyester & $2-13$ & \multirow[t]{2}{*}{731} & & \\
\hline & & Teflan & Epoxy & 2,10 & & & \\
\hline \multirow[t]{2}{*}{$\triangle$} & \multirow[t]{2}{*}{$\begin{array}{c}\text { Lapp et al } \\
1958\end{array}$} & Folyurethane & $\begin{array}{l}\text { Epoxy } \\
\text { Aluinge } \\
\text { Polyester }\end{array}$ & $\begin{array}{l}15-30 \\
8 * 11 \\
10\end{array}$ & \multirow[t]{2}{*}{731} & \multirow[t]{2}{*}{1} & \multirow[t]{2}{*}{1.9} \\
\hline & & Tellor & $\begin{array}{l}\text { Epoxy } \\
\text { Aluelaum }\end{array}$ & $\begin{array}{l}10 \\
62.3\end{array}$ & & & \\
\hline & \multirow[t]{2}{*}{$\underset{1967}{\text { Schatet et }}$} & Alu=inue & $\begin{array}{l}\mathrm{AlPO}_{4} \\
\mathrm{PBI} \\
\text { Polyalde } \\
\text { Epoxy }\end{array}$ & $\begin{array}{l}20 \\
20.40 \\
30 \\
30 \\
\end{array}$ & $\begin{array}{l}1596 \\
2360 \\
3169\end{array}$ & \multirow[t]{2}{*}{ i.s } & \multirow[t]{2}{*}{1.9} \\
\hline & & $\begin{array}{l}\text { Gaca } \\
\text { Polyurethate } \\
\text { Ieflon } \\
\text { Nicke I }\end{array}$ & Epoxy & $\begin{array}{l}20 \\
26,30 \\
35 \\
10\end{array}$ & & & \\
\hline & \multirow[t]{5}{*}{$\begin{array}{r}\text { Schelett } \\
2970\end{array}$} & Aluesina & $\begin{array}{l}\text { Poly=1de } \\
\text { Epoxy }\end{array}$ & $\begin{array}{l}10 \\
40\end{array}$ & $\begin{array}{l}1596 \\
2360 \\
2731 \\
3159\end{array}$ & \multirow[t]{5}{*}{2.5} & \multirow[t]{5}{*}{1.9} \\
\hline & & Gaco & Epoxy & 10 & $\begin{array}{l}1596 \\
3159 \\
\end{array}$ & & \\
\hline & & Grethane & & $10,15,20,30$ & $\begin{array}{l}1536 \\
2350 \\
\end{array}$ & & \\
\hline & & Folycthy lene & Epoxy & 30 & \multirow[t]{2}{*}{2151} & & \\
\hline & & Nickel & Folyosde & 12 & & & \\
\hline & \multirow{2}{*}{$\begin{array}{l}\text { Schettet } \\
1971\end{array}$} & Urethane & Alvelne & 15 & \multirow[t]{2}{*}{131} & \multirow[t]{2}{*}{1} & \multirow[t]{2}{*}{1.9} \\
\hline & & Neoptene & $\begin{array}{l}\text { Epoxy } \\
\text { Alurinuta }\end{array}$ & 22 & & & \\
\hline
\end{tabular}

* Material properties used in obtaining lïgs. 9-11 are from References (6), (9)-(10).

$\rho$ is the density of the material undergoing erosion.

For homogeneous materials the values of $a_{3}$ and $a_{6}$ were determined by Springer and Baxi [13] and were found to be $a_{3}=0.023$ and $a_{6}=0.7$. Similarly as for the incubation period, we adopt the same values of these constants for the present problem of homogeneous substrates covered by a single layer of coating, i.e.

$$
\alpha^{*}=0.023 \frac{1}{\left(1_{i}^{*}\right)^{0.7}}
$$


In the case of $\bar{k} \rightarrow 0$ and/or $\psi_{s c} \rightarrow 0$ the incubation period $n_{i}^{*}$ reduces to $n_{i H}^{*}$ (see Section IV). Consequently, under these conditions, $\alpha^{*}$ (given by Equation 54) becomes the same as given by Springer and Baxi's formula for homogeneous materials.

The validity of the foregoing model was assessed by comparing $\alpha^{*}$, calculated by Equation (54) to available experimental data. This comparison, given in Figure 10, shows very good agreement between the calculated and measured $\alpha^{*}$ values. This lends further confidence to the model.

\section{TOTAL MASS LOSS}

The total mass loss was given by Equation (4b) as

$$
m=\alpha\left(n-n_{i}\right)
$$

Introducing the dimensionless parameter

$$
m^{*}=\frac{m}{\rho_{c} d}
$$

Equations (4b), (47) and (53) yield

$$
m^{*}=\alpha^{*}\left(n^{*}-n_{i}^{*}\right) \quad \text { or } \quad \frac{m^{*}}{\alpha^{*}}=n^{*}-n_{i}^{*}
$$

According to Equation (56) it should be possible to correlate all erosion data on a $m^{*} / \alpha^{*}$ versus $\left(n^{*}-n_{i}^{*}\right)$ plot. Therefore, we have included all the existing data on such a plot (Figure 11). In this figure the theoretical result given by our model (Equation 56) is also indicated. The agreement between the model and the data is quite good, particularly in view of the large errors inherent in many of the measurements.

\section{FATIGUE FAILURE OF THE SUBSTRATE}

The foregoing analysis was based on the assumption that the coating fails before the substrate. Under some conditions, however, the substrate may fail before the coating. The analyses presented in Sections IV, V and VI can be applied readily to such a situation. To calculate the behavior and failure of the substrate only minor modifications need be made in the previous results. The average stress at the surface of the coating $\bar{\sigma}^{\circ}$ (Equation 25a) must be replaced by the average stress at the coat-substrate interface $\bar{\sigma}^{h}$ (Equation 25b). Consequently, Equation (49) must be written as

$$
n_{i}^{*}=a_{1}\left(\frac{S_{e}}{\bar{\sigma}^{h}}\right)^{a_{2}}
$$




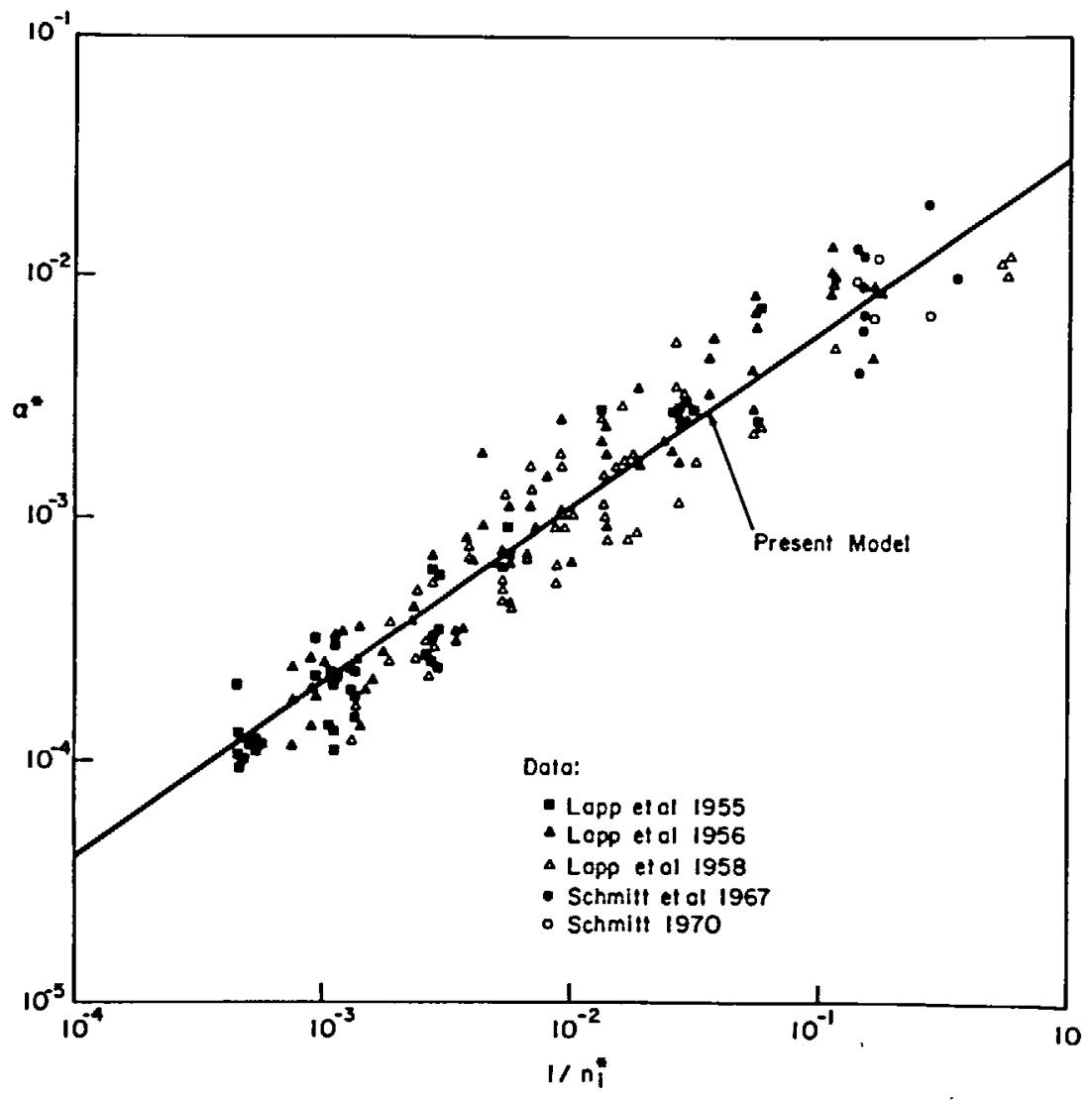

Figure 10. Rate of Erosion Versus the Imerse of the Incubation Period. Solid Line: Model (Equation 54). Sy'mbols Dcfincd in Table 1.

Furthermore, in calculating $S_{e}$ (Equations 45,46$)$ the parameters $\left(\sigma_{u_{c}}\right),\left(\sigma_{I_{c}}\right)$ and $\nu_{c}$ must be replaced by the properties of the substrate $\left(\sigma_{u_{s}}\right),\left(\sigma_{I_{s}}\right)$ and $v_{s}$. All other results remain unaltered.

\section{LIMITS OF APPLICABILITY OF MODEL}

The results presented in Sections II-VI are valid when (a) there is a finite incubation period, and (b) the mass loss varies linearly either with time $t$ or with the number of impacts $n$. The first of this condition is met when the following inequality is satisfied

$$
n_{i}^{*}>1
$$

According to Equation (51) this condition may also be expressed as 


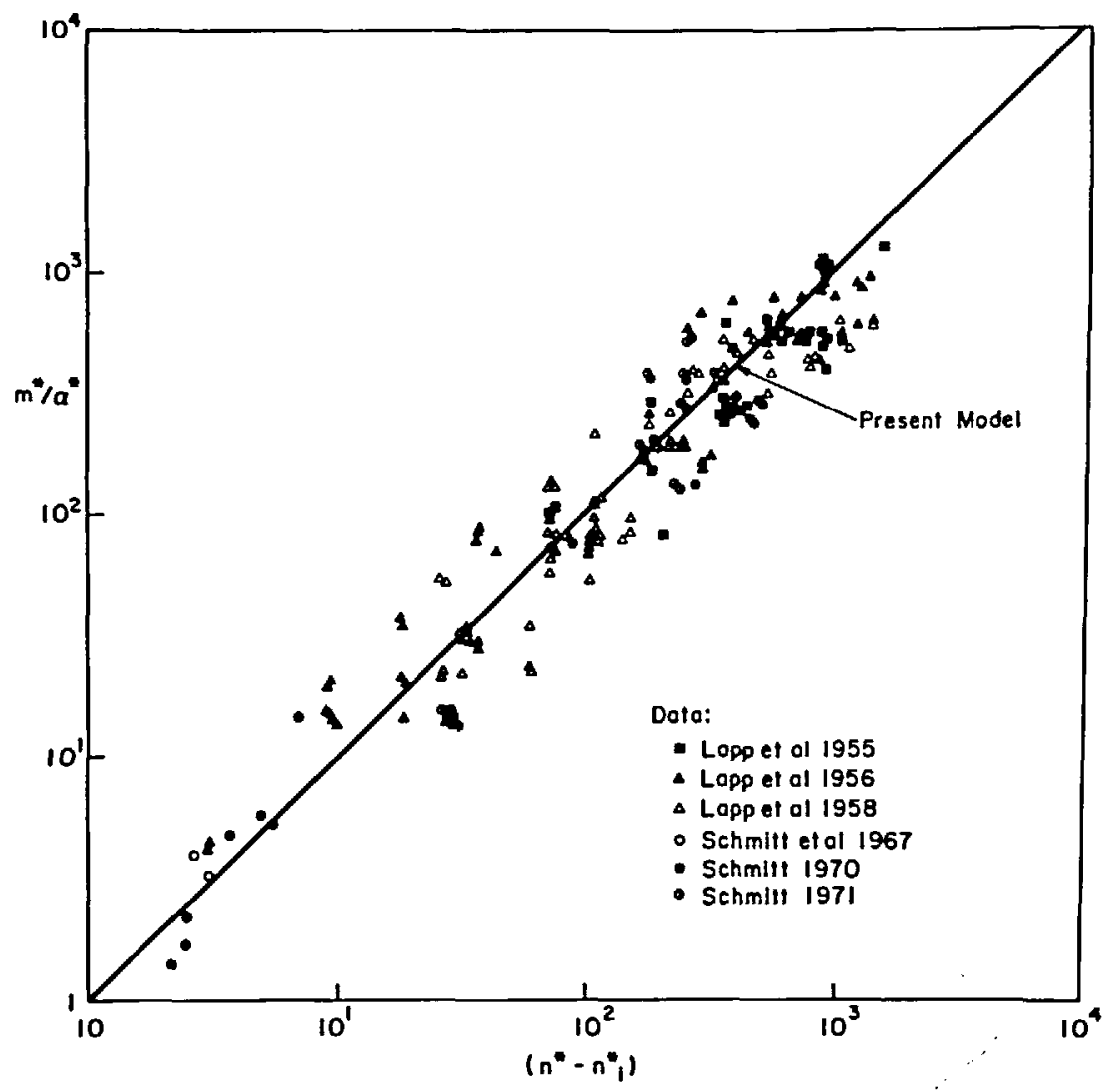

Figure 11. Comparison of Present Mfodel (Solid Line, Equation 56) with Experimental Results. Symbols Defined in Table 1.

$$
S_{e} / \vec{\sigma}_{o}>8
$$

Equations (58a) or (58b) provide the lower limit of the applicability of the model. The upper limit beyond which the present model cannot be applied is determined by the second condition given above, namely that the mass loss must vary linearly with $t$ or $n$. An estimate of this limit was made by observing that up to about $n=3 n_{i}$ the data obtained at various values of $n$ did not show any systematic deviation from the model. Thus, the results are valid as long as the number of impacts is less than three times the incubation period, i.e.

$$
n^{*}<3 n_{i}^{*}
$$

Using Equation (51) we obtain the following expression for the upper limit 


$$
n<21.3 \times 10^{6}\left(\frac{S_{\mathcal{E}}}{\bar{\sigma}^{o}}\right)
$$

Note that the two limits expressed by Equations (58) and (59) do not impose any constraints on either the material or the impact velocity. Thus, the results are valid for any material and for any impact velocity, provided that the experimental conditions fall within the range specified by Equations (58) and (59).

\section{ACKNOWLEDGMENTS}

The authors wish to thank Mr. G. F. Schmitt, Jr. for his valuable comments and for providing many of the references and data used in this investigation.

This work was supported by U.S. Air Force Contract F33615-72-C-1563, and was administered by the Air Force Materials Laboratory, Air Force Systems Command, Wright-Patterson Air Force Base, Dayton, Ohio.

\section{NOMENCLATURE}

\begin{tabular}{|c|c|}
\hline$a_{1}-a_{6}$ & constants (dimensionless) \\
\hline$A$ & area $\left(\mathrm{ft}^{2}\right)$ \\
\hline$b$ & constant defined by Equation (43) (dimensionless) \\
\hline$b_{2}$ & knee in the fatigue curve \\
\hline$C$ & speed of sound $(\mathrm{ft} / \mathrm{sec})$ \\
\hline$d$ & diameter of the droplet $(\mathrm{ft})$ \\
\hline$E$ & modulus of elasticity $\left(\mathrm{lbf} / \mathrm{ft}^{2}\right)$ \\
\hline$f$ & number of stress cycles (see Equation 31) \\
\hline$F$ & force (lbf) \\
\hline$h$ & thickness of coat $(\mathrm{ft})$ \\
\hline$I$ & rain intensity $(\mathrm{ft} / \mathrm{sec})$ \\
\hline$k_{e}$ & $\begin{array}{l}\text { number of stress wave reflections in the coating } \\
\text { required for the stress at coat-substrate interface } \\
\text { to reach a value of } 63.3 \text { percent of } \sigma_{\infty} \\
\text { (dimensionless) }\end{array}$ \\
\hline$k_{L}$ & $\begin{array}{l}\text { total number of stress wave reflections in the coating } \\
\text { during the impact period (dimensionless) }\end{array}$ \\
\hline $\bar{k}$ & $\begin{array}{l}\text { average number of stress wave reflections in the } \\
\text { coating (dimensionless) }\end{array}$ \\
\hline$m$ & mass eroded per unit area $\left(\mathrm{lbm} / \mathrm{ft}^{2}\right)$ \\
\hline$m^{*}$ & dimensionless mass loss defined by Equation (55) \\
\hline$n$ & number of drops impinging per unit area (number $/ \mathrm{ft}^{2}$ ) \\
\hline$n^{*}$ & $\begin{array}{l}\text { number of drops impinging per site, see Equation (47) } \\
\text { (dimensionless) }\end{array}$ \\
\hline
\end{tabular}


fatigue life (dimensionless)

stress $\left(\mathrm{lbf} / \mathrm{ft}^{2}\right)$

distance $(\mathrm{ft})$

parameter defined by Equation (45) $\left(\mathrm{lbf} / \mathrm{ft}^{2}\right)$

parameter defined by Equation (46) (lbf/ $\mathrm{ft}^{2}$ )

time (sec)

time required for $k_{e}$ number of stress wave reflections to take place in the coating ( $\mathrm{sec}$ )

the duration of impact (sec)

velocity of impact $(\mathrm{ft} / \mathrm{sec})$

terminal velocity of a rain droplet $(\mathrm{ft} / \mathrm{sec})$

weight loss due to erosion (lbf)

dynamic impedance $\left(\mathrm{lbm} /\left(\mathrm{ft}^{2}-\mathrm{sec}\right)\right)$

\section{GREEK LETTERS}

$\alpha$

rate of mass loss (lbm/impact)

(see Figure 2b)

dimensionless rate of mass loss

(see Equation 53)

the ratio of $k_{L}$ to $k_{e}\left(=k_{L} / k_{e}\right)$

Poisson's ratio (dimensionless)

density $\left(\mathrm{lbm} / \mathrm{ft}^{\mathbf{3}}\right)$

angle (radians)

stress $\left(\mathrm{lbf} / \mathrm{ft}^{2}\right)$

stress amplitude $\left(\mathrm{lbf} / \mathrm{ft}^{2}\right)$

equivalent dynamic stress defined by

Equation (33) $\left(\mathrm{lbf} / \mathrm{ft}^{2}\right)$

mean stress (lbf/ $\left./ \mathrm{ft}^{2}\right)$

mean stress after $k_{L}$ number of stress wave

reflections ( $\left.\mathrm{lbf} / \mathrm{ft}^{2}\right)$

endurance limit $\left(\mathrm{lbf} / \mathrm{ft}^{2}\right)$

ultimate tensile strength $\left(\mathrm{lbf} / \mathrm{ft}^{2}\right)$

parameter defined by Equation (8)

\section{SUBSCRIPTS}

$c$
$i$
$f$
$k$
$L$

coating

end of incubation period

upper limit of validity of model

the number of stress wave reflections in the coating

liquid 
solid

coat-substrate interface

Lc liquid-coat interface

\section{SUPERSCRIPTS}

$h$

$o$ coat-substrate interface

liquid-coat interface

\section{REFERENCES}

1. R. R. Lapp, R. H. Stutzman, N. E. Wahl, "A Study of the Rain Erosion of Plastic and Metals," WADC Technical Report 53-185, Part 2. Wright-Patterson Air Force Base, Dayton, Ohio. May 1955.

2. R. R. Lapp, R. H. Stutzman, N. E. Wahl, "Summary Report on the Rain Erosion of Aircraft Material," WADC Technical Report 53-185, Part 3. Wright-Patterson Air Force Base, Dayton, Ohio. Sept. 1956.

3. R. R. Lapp, D. H. Thorpe. R. H. Stutzman, N. E. Wahl, "The Study of Erosion of Aircraft Materials at High Speed in Rain," WADC Technical Report 53-185, Part 4. Wright-Patterson Air Force Base, Dayton, Ohio. May 1958.

4. G. F. Schmitt, G. J. Tatnall, K. W. Foulke, "Joint Air Force-Navy Supersonic Rain Erosion Evaluations of Materials," AFML-TR-67-164. Air Force Materials Laboratory, WrightPatterson Air Force Base, Dayton, Ohio. 1967.

5. G. F. Schmitt, "Research for Improved Subsonic and Supersonic Rain Erosion Resistant Material," AFML-TR-67-211, Air Force Materials Laboratory, Wright-Patterson Air Force Base, Dayton, Ohio. January 1968.

6. G. F. Schmitt, "Erosion Bchavior of Polymeric Coatings and Composites at Subsonic Velocities," Proceedings of the Third International Conference on Rain Erosion and Associated Phenomena," (Edited by A. A. Fyall and R. B. King). Royal Aircraft Establish. ment, England. August, 1970, p. 107.

7. J. W. Morris, "Supersonic Rain and Sand Erosion Research," AFML-TR-69-287, Part II. "Mechanic Investigation of Rain Erosion," Air Force Materials Laboratory, Wright-Patterson Air Force Base, Dayton, Ohio. September 1969.

8. O. G. Engel, A. J. Piekutowski, "Investigation of Composite-Coating Systems for RainErosion Protection," Prepared under contract N00019-71-C-0108 for Naval Air Systems Command, Department of the Navy, at the University of Dayton, Research Institute, Dayton, Ohio.

9. A. F. Conn, "Research of Dynamic Response and Adhesive Failures of Rain Erosion Resistant Coating," Technical Report 811-1 Hydronautics, Laurel, MD. January 1969.

10. A. F. Conn, "Prediction of Rain Erosion Resistance from Measurements of Dy'namic Properties," Technical Paper, Hydronautics, Laurel, MD. April 1970.

11. A. F. Conn, S. L. Rudy, "Further Research on Predicting the Rain Erosion Resistance of Material," Teclinical Report 7107-1, Hydronautics, Laurel, MD. May 1972.

12. C. H. Mok, "A Cumulative Damage Concept in Rain Erosion Studies," AISS Journal, Vol. 7 (1969), p. 751.

13. G. S. Springer, C. B. Baxi, "A Model for Rain Erosion of Homogeneous Material," Technical Report AFML-TR-72-106, Air Force Matcrials Laboratory, Wright-Patterson Air Force Base, Dayton, Ohio. June 1972: also, ASTM Symposium on Lirosion, Wear and Interfaces with Corrosion. Philadelphia, June 1973. (Proceedings in print). 
14. F. J. Heymann, "Erosion by Cavitation, Liquid Impingement and Solid Impingement: A Review," Engineering Report E-1460, Westinghouse Electric Corporation, Lester, Pennsylvania, March 1968.

15. F. J. Heymann, "On the Shock Wave Velocity and Impact Pressure in High Speed LiquidSolid Impact," Trans. ASME, J. Basic Engineering, Vol. 90, (1968), p. 400.

16. H. Kolsky, Stress Waves in Solids, Dover Publications, Inc., New York (1963).

17. G. S. Springer, C. I. Yang and P. S. Larsen, "Analysis of Rain Erosion of Coated Materials," Technical Report AFML-TR-73-227, Air Force Materials Laboratory, Wright-Patterson Air Force Base, Dayton, Ohio, September 1973.

18. J. H. Brunton, "Liquid Impact and Material Removal Phenomena," Technical Memorandum No. 33-354, Jet Propulsion Laboratory, California Institute of Technology, Pasadena, California, June 1964.

19. F. J. Heymann, "A Survey of Clues to the Relation Between Erosion Rate and Impingement Conditions," Proceedings of the Second Meersburg Conference on Rain Erosion and Allied Phenomena, (edited by A. A. Fyall and R. B. King), Royal Aircraft Establishment, Farnborough, England, August 1967, p. 685.

20. W. C. Leith, A. L. Thompson, "Some Corrosion Effects in Accelerated Cavitation Damage," Trans. ASME, J. Basic Engineering, Vol. 82D (1960), p. 795.

21. R. Mathieson, J. M. Hobbs, "Cavitation Erosion: Comparative Tests," Engineering, Vol. 189 (1960), p. 136.

22. J. F. Ripken, "A Testing Rig for Studying Impingement and Cavitation Damage," in Erosion by Cavitation on Impingement, ASTM STP 408, American Society for Testing and Materials, 1967, p. 3.

23. A. Thiruvengadam, S. L. Rudy, M. Gunasekaran, "Experimental and Analytical Investigations on Liquid Impact Erosion," in Characterization and Determination of Erosion Resistance, ASTM STP 474, American Society for Testing and Materials, (1970), p. 249.

24. M. A. Miner, "Cumulative Damage in Fatigue," J. Appl. Mlech., Vol. 12, (1945), p. Al59.

25. S. Timoshenko, Theory of Elasticity, McGraw-Hill Book Co., New York, 1934.

26. R. C. Juvinall, Stress, Strain and Strength, McGraw-Hill Book Co., New York, 1967. 